\title{
Nonlinear excitations in two-dimensional molecular structures with impurities
}

\author{
Gaididei, Yuri Borisovich; Rasmussen, Kim; Christiansen, Peter Leth
}

Published in:

Physical Review E. Statistical, Nonlinear, and Soft Matter Physics

Link to article, DOI:

10.1103/PhysRevE.52.2951

Publication date:

1995

Document Version

Publisher's PDF, also known as Version of record

Link back to DTU Orbit

Citation (APA):

Gaididei, Y. B., Rasmussen, K., \& Christiansen, P. L. (1995). Nonlinear excitations in two-dimensional molecular structures with impurities. Physical Review E. Statistical, Nonlinear, and Soft Matter Physics, 52(3), 2951-2962. https://doi.org/10.1103/PhysRevE.52.2951

\section{General rights}

Copyright and moral rights for the publications made accessible in the public portal are retained by the authors and/or other copyright owners and it is a condition of accessing publications that users recognise and abide by the legal requirements associated with these rights.

- Users may download and print one copy of any publication from the public portal for the purpose of private study or research

- You may not further distribute the material or use it for any profit-making activity or commercial gain

- You may freely distribute the URL identifying the publication in the public portal 


\title{
Nonlinear excitations in two-dimensional molecular structures with impurities
}

\author{
Yu. B. Gaididei, ${ }^{1}$ K. Ø. Rasmussen, ${ }^{2}$ and P. L. Christiansen ${ }^{2}$ \\ ${ }^{1}$ Institute for Theoretical Physics, Metrologicheskaya Street 14 B, 252143 Kiev 143, Ukraine \\ ${ }^{2}$ Institute of Mathematical Modelling, Technical University of Denmark, DK-2800 Lyngby, Denmark
}

(Received 30 December 1994; revised manuscript received 11 April 1995)

\begin{abstract}
We study the nonlinear dynamics of electronic excitations interacting with acoustic phonons in twodimensional molecular structures with impurities. We show that the problem is reduced to the nonlinear Schrödinger equation with a varying coefficient. The latter represents the influence of the impurity. Transforming the equation to the noninertial frame of reference coupled with the center of mass we investigate the soliton behavior in the close vicinity of the impurity. With the help of the lens transformation we show that the soliton width is governed by an Ermakov-Pinney equation. We also investigate bound state of the soliton with impurity and show that in addition to the radially symmetric bound state a dipolelike state can exist if the number of excitations in the soliton does not exceed some threshold value. We study both the equilibrium states and the dynamics of the dipolelike excitations. Analytical results are in good agreement with numerical simulations of the nonlinear Schrödinger equation.
\end{abstract}

PACS number(s): 87.15. $-\mathrm{v}$, 68.55.Ln, 34.30. $+\mathrm{h}, 33.90 .+\mathrm{h}$

\section{INTRODUCTION}

The study of energy transfer in various types of mesoscopic molecular structures, from molecular clusters and small molecular aggregates ( $J$ aggregates) to thin molecular films (Langmuir-Blodgett films) is an area of intense current interest. The field derives its importance from being a part of the general area of energy transfer in condensed matter [1,2] and from its connection with biological problems such as photosynthesis $[3,4]$, muscle contraction [5], etc. Langmuir-Blodgett (LB) films of dyes have also found technological applications as sensitizers in the photographic industry [6] and in photoelectronics [7]. LB molecular films with their well determined molecular arrangements are particularly suited to energy transfer studies (see review articles by Kuhn, Möbius, and Bucher [8], Blinov [9], and Tredgold's monograph [10]). Scheibe aggregates are specially ordered compact molecular LB structures of dyes [11] that manifest themselves as redshifted narrow bands in the absorption spectrum, which were first discovered by Scheibe [12] and Jelly [13]. Studying the fluorescence of a monolayer of oxacyanine dye doped with a thiacyanine dye, Möbius and Kuhn observed $[14,15]$ at room temperature very efficient electronic excitation energy transfer from host molecules to acceptor molecules. The host fluorescence is effectively quenched by guest molecules even at a molar ratio host:acceptor of $10^{4}$. In Ref. [14], hopping and coherent excitons are discussed as possible mechanisms for energy transfer and it is found that the coherent exciton approach provides an adequate description of the experimental results. The crucial role of the coherent properties of the electronic excitations in small molecular aggregates was emphasized in Refs. [16,17].

To explain the highly efficient energy transfer in LB monolayers, two theoretical models were proposed. A pure quantum-mechanical approach to the problem has recently been advocated in a series of papers [18]. It was assumed that the exciton-phonon interaction is weak and may be treated as a perturbation. The quantummechanical calculations were carried out both for oneand two-dimensional molecular assemblies and it was shown that the planar geometry of LB-Scheibe aggregates plays a special role in the remarkable efficiency of energy transfer. Huth, Gutmann, and Vitiello [19] proposed a nonlinear dynamical model for energy transfer in LBScheibe aggregates. They assumed that in the system under consideration the exciton-phonon interaction is strong and reduced the problem to the nonlinear Schrödinger equation (NLS). The model was formulated in one dimension where the NLS equation has soliton solutions. Christiansen, Pagano, and Vitiello [20] investigated the model in the case of two spatial dimensions using ring wave solutions [21]. It is known (see, e.g., [22]) that the two-dimensional NLS does not have stable soliton solutions: Nonlinear excitations either collapse or disperse. In Ref. [20] ring solitons were considered and their collapse time was identified with the exciton lifetime in Möbius and Kuhn's experiments. Temperature effects in the nonlinear model of monolayer Scheibe aggregates were studied quite recently by Bang et al. [23]. Thermal fluctuations of the phonons were included and the resulting equation for the excitations was the two-dimensional NLS equation with noise. The temperature-dependent nonlinear coherence time (i.e., the time during which a nonlinear excitation maintains its shape) was estimated from numerical simulations. A temperature interval where this time is in correspondence with the lifetime of the Möbius and Kuhn excitons was found (see also $[24,25])$.

It is worth stressing that while the nonlinear model developed in Refs. $[19,20]$ is intended to explain an energy transfer from host molecules to impurity (acceptor) molecules, the model does not directly take impurity effects into account. But impurities can drastically influence the soliton dynamics. In the one-dimensional 
case, it was shown both for the continuum NLS equation (see the review paper [26]) and for its discrete analog [27].

The purpose of the present paper is to investigate the behavior of the nonlinear excitations in two-dimensional molecular structures with impurities. In Sec. II we present a model and derive equations of motion for the electronic excitations. Using the approach that was proposed in Ref. [23], we show that the dynamics of the electronic excitations in molecular system with impurities is governed by the two-dimensional NLS equation with a linear potential. The former describes the influence of the impurity molecule. Section III deals with soliton dynamics in the close vicinity of the impurity. We consider the impurity potential in the parabolic approximation and show that a lens transformation can reduce the problem to an Ermakov-Pinney differential equation. It is shown that the analytical results are in good agreement with the results of numerical simulations that were carried out for the NLS equation. Section IV is devoted to an investigation of a new dipolelike stationary state that arise as the result of interaction between the nonlinear excitations and the impurity. To study analytically these stationary states, we use the method of collective coordinates and check the results by means of numerical simulations. Qualitative agreement between both approaches was found. In Sec. $\mathrm{V}$ the model parameters are estimated for the case of oxacyanine dye monolayer. The relevance of nonlinear excitations is shown.

\section{MODEL AND EQUATIONS OF MOTION}

Let us consider a two-dimensional molecular system in which one of the sites is occupied by an impurity molecule. We shall assume that the impurity molecules and host molecules are sterically very similar. Therefore, the impurity molecule substituting for the host molecule does not distort significantly the lattice in which the host molecules are arranged.

We shall investigate nonlinear excitation dynamics in this two-dimensional molecular system and assume that the density of excitations is low and that the nonlinear character of the excitation dynamics is caused by an exciton-phonon interaction. The derivation of the nonlinear equations of motion is a straightforward generalization of that for an ideal molecular system (see, e.g., [23] and the Appendix for details). As a result, we obtain that the excitation dynamics is governed by the following effective Hamiltonian:

$$
H=\sum_{\vec{n}}\left\{E_{\vec{n}}\left|\phi_{\vec{n}}\right|^{2}-\frac{1}{2} V\left|\phi_{\vec{n}}\right|^{4}-\sum_{\vec{n}} J_{\vec{n} \vec{n}} \phi_{\vec{n}}^{*} \phi_{\overrightarrow{n^{\prime}}}\right\} .
$$

Here, $\phi_{\vec{n}}(t)$ is the excitation function of the molecule at site $\vec{n}, J_{\vec{n} \vec{n}}$ is the matrix element of the excitation transition from the molecule $\vec{n}$ to the molecule $\vec{n}^{\prime}$, and $V$ is the nonlinearity parameter. $E_{\vec{n}}$ is the on-site excitation energy. It depends on the molecule position $\vec{n}$ in the layer and may be represented in the form

$$
E_{\vec{n}}=\left(E_{a}-E_{h}\right) \delta_{\vec{n}, 0}+\Delta_{\vec{n}}
$$

Here $E_{a}-E_{h}$ is the difference between excitation ener- gies of the impurity molecule $\left(E_{a}\right)$ and the host molecule $\left(E_{h}\right)$. We assume that the impurity is placed at the zeroth site. The second term in Eq. (2.2) characterizes the change in the host excitation energy caused by the presence of an impurity in the system. Its general form is given by

$$
\begin{aligned}
\Delta_{\vec{n}}= & \left\langle\psi_{\vec{n}, e} \widetilde{\psi}_{\overrightarrow{0}, g}|W| \widetilde{\psi}_{\overrightarrow{0}_{g}} \psi_{\vec{n}, e}\right\rangle-\left\langle\psi_{\vec{n}, e} \psi_{\overrightarrow{0}, g}|W| \psi_{\overrightarrow{0}, g} \psi_{\vec{n}, e}\right\rangle \\
& -\left\langle\psi_{\vec{n}, g} \widetilde{\psi}_{\overrightarrow{0}, g}|W| \widetilde{\psi}_{\overrightarrow{0}, g} \psi_{\vec{n}, g}\right\rangle+\left\langle\psi_{\vec{n}, g} \psi_{\overrightarrow{0}, g}|W| \psi_{\overrightarrow{0}, g} \psi_{\vec{n}, g}\right\rangle,
\end{aligned}
$$

where $\left\langle\psi_{\vec{n}, \lambda} \psi_{\vec{n}^{\prime}, \lambda^{\prime}}|W| \psi_{\vec{n}, \lambda} \psi_{\vec{n}, \lambda^{\prime}}\right\rangle$ is the matrix element of the interaction between sites $\vec{n}$ and $\vec{n}^{\prime}$, one of which is in the state described by the wave function $\psi_{\vec{n}, \lambda}$ and the other in the state $\psi_{n^{\prime}, \lambda^{\prime}} . \lambda=e(\lambda=g)$ corresponds to the excited (ground) state of the molecule, $\widetilde{\psi}_{0, g}$ is the wave function of the ground state of the impurity. It is seen that the absolute value of $\Delta_{\vec{n}}$ has a maximum at $\vec{n}=\overrightarrow{0}$ and $\Delta_{\vec{n}} \rightarrow 0$ if $|\vec{n}| \rightarrow \infty$. The general expression for $\Delta_{\vec{n}}$ is very complicated because the interactions of different types (dipole-dipole, dipole-quadrupole, quadrupolequadrupole, etc.) contribute to it. The contribution $\Delta_{\vec{n}}^{\mathrm{dd}}$ of the dipole-dipole interaction looks the simplest:

$$
\begin{aligned}
& \Delta_{\vec{n}}^{\mathrm{dd}}=\frac{1}{|\vec{n}|^{3}}\left\{\left[\vec{d}_{h}(\mathrm{ex})-\vec{d}_{h}(\mathrm{gr})\right] \cdot\left[\vec{d}_{i}(\mathrm{gr})-\vec{d}_{h}(\mathrm{gr})\right]\right. \\
&-3\left[\frac{\vec{n}}{|\vec{n}|}, \vec{d}_{h}(\mathrm{ex})-\vec{d}_{h}(\mathrm{gr})\right] \\
&\left.\times\left\{\frac{\vec{n}}{|\vec{n}|}, \vec{d}_{h}(\mathrm{ex})-\vec{d}_{h}(\mathrm{gr})\right]\right\},
\end{aligned}
$$

where $\vec{d}_{h}(\mathrm{gr})\left[\vec{d}_{i}(\mathrm{gr})\right]$ is the dipole moment of the host (impurity) molecule in the ground electronic state. $\vec{d}_{h}(\mathrm{ex})$ is the dipole moment of the host molecule in the excited electronic state. We see that the magnitude and sign of $\Delta \vec{n}$ dd depend not only on the characteristics of the electronic states $\left[\vec{d}_{h, i}(\mathrm{gr}), \vec{d}_{h}(\mathrm{ex})\right]$ but also on the arrangement of the molecules in the system. We shall assume that different types of intermolecular interactions acting together make the function $\Delta_{\vec{n}}$ smoother and in what follows we shall consider $\Delta_{\vec{n}}$ as a function of the distance $|\vec{n}|$ from the impurity.

The Hamiltonian Eq. (2.1) conserves the number of excitations in the crystal. We shall assume that there is only one excitation. Thus the normalization condition for the excitation function $\phi_{\vec{n}}(t)$ becomes

$$
\sum_{\vec{n}}\left|\phi_{\vec{n}}(t)\right|^{2}=1
$$

The equations of motion for $\phi_{\vec{n}}(t)$ are

$$
i \hbar \dot{\phi}_{\vec{n}}+\sum_{\vec{n}} J_{\vec{n} \vec{n}^{\prime}} \phi_{\vec{n}}+V\left|\phi_{\vec{n}}\right|^{2} \phi_{\vec{n}}=E_{\vec{n}} \phi_{\vec{n}}
$$

Thus the discrete self-trapping (DST) equation, which takes into account inhomogeneity effects, is obtained. It is well known that even in the one-dimensional case the 
interplay between nonlinearity and inhomogeneity makes the DST problem very complex and difficult [27]. In the case of two-dimensional aggregate, the discrete equations (2.6) are impractical to study. Therefore, we restrict ourselves to the continuum limit of Eq. (2.6). Taking only the nearest neighbor excitation transfer $J$ into account and making the gauge transformation $\phi_{\vec{n}} \rightarrow \phi_{\vec{n}} \exp (i z J t$ $/ \hbar)$ ( $z$ is the number of nearest neighbors), Eq. (2.6) may be approximated by the two-dimensional nonlinear Schrödinger equation (NLS) with a spatially variable coefficient

$$
i \hbar \phi_{t}+\frac{J z}{2} l^{2} \nabla^{2} \phi+l^{2} V|\phi|^{2} \phi=E(\vec{r}) \phi,
$$

where $|\phi(\vec{r}, t)|^{2}=\left|\phi_{\vec{n}}\right|^{2} / l^{2}$ is a probability density

$$
\int d \vec{r}|\phi(\vec{r}, t)|^{2}=1
$$

and $l$ is the lattice constant. $E(\vec{r})$ is the continuum limit of the on-site excitation energy $E_{\vec{n}}$. It determines an energetic profile for an excitation in the vicinity of the impurity molecule. As mentioned above, in real systems this profile may have a very complicated form. It depends on the symmetry of the lattice and on the type of excitation (singlet, triplet, dipole active or quadrupole active, etc.). In the present paper we want to consider some general features of soliton dynamics in the vicinity of an impurity molecule. Therefore, for the sake of simplicity we shall assume that $E(\vec{r})$ is an axially symmetric Gaussian function

$$
E(r)=E \exp \left[-\frac{r^{2}}{r_{0}^{2}}\right),
$$

where $E$ is the strength of the impurity and $r_{0}$ is the radius.

Introducing dimensionless variables $\vec{\rho}=\vec{r} / r_{0}$, $\tau=\left(z l^{2} J t\right) /\left(2 r_{0}^{2} \hbar\right)$, and $\psi=\left[\left(2 V r_{0}^{2}\right) /(J z)\right]^{1 / 2} \phi$, Eqs. (2.7) and (2.9) are replaced by

$$
\begin{aligned}
& i \psi_{\tau}+\nabla^{2} \psi+|\psi|^{2} \psi=U(\rho) \psi, \\
& U(\rho)=\epsilon \exp \left(-\rho^{2}\right),
\end{aligned}
$$

where the dimensionless parameter $\epsilon=\left(2 E r_{0}^{2}\right) /\left(z J l^{2}\right)$ characterizes the power of the impurity and

$$
\int d \vec{\rho}|\psi|^{2}=\frac{2 V}{z J} \equiv N
$$

The two-dimensional NLS equation is not integrable and possesses unstable solutions that may either disperse or collapse. These two types of solutions are separated by the so-called ground state (GS) solution whose width does not change in time. The GS solution [to Eq. (2.10) with $U \equiv 0]$, approximately given by [28], is

$$
\psi_{s}=A_{s} \operatorname{sech}\left(\frac{\rho}{B_{s}}\right) e^{i \tau}
$$

in the case of zero initial velocity and center at $\rho=0$. The GS amplitude $A_{s}$ and the GS width $B_{s}$ are given by

$$
\begin{aligned}
& A_{s}=\left(\frac{12 \ln 2}{4 \ln 2-1}\right)^{1 / 2}, \\
& B_{s}=\left[\frac{2 \ln 2+1}{6 \ln 2}\right]^{1 / 2} .
\end{aligned}
$$

Insertion of Eqs. (2.13), (2.14a), and (2.14b) into Eq. (2.12) yields

$$
N=N_{s}=11.7 \text {. }
$$

For initial conditions, $\psi=\psi(\rho, 0)$, with $N$ larger (smaller) than the $N_{s}$ the solution, $\psi=\psi(\rho, \tau)$ of the twodimensional NLS equation, collapses in finite time (disperses).

\section{SOLITON MOTION IN THE CLOSE VICINITY OF IMPURITY}

Let us consider the motion of a soliton in the neighborhood of the impurity. We shall assume that the radius $r_{0}$ of the impurity is large compared with the width of the soliton. In this case we can expand the impurity potential $U(\rho)$, keeping only terms of second order $U(\rho) \simeq \epsilon\left(1-\rho^{2}\right)$ and in this way the problem reduces to the investigation of soliton motion in a parabolic potential. Therefore, we shall consider the soliton motion in two-dimensional parabolic potential

$$
U(\rho, \tau)=-\epsilon(\tau) \rho^{2},
$$

where $\epsilon(\tau)$ is an arbitrary function of time. The soliton motion in the one-dimensional parabolic potential was investigated by Chen and Liu [29] and quite recently by Nogami and Toyama [30].

Introducing the transformation to the noninertial frame of reference in which the center of mass of the excitation is at rest,

$$
\psi(\vec{\rho}, \tau)=\varphi(\vec{\rho}, \tau) \exp \left\{\frac{i}{2} \dot{\vec{R}} \cdot \vec{\rho}-i \int_{0}^{\tau} d \widetilde{\tau}\left\{\frac{1}{4}\left[\dot{\vec{R}}(\widetilde{\tau})^{2}+\epsilon(\widetilde{\tau}) \vec{R}^{2}(\widetilde{\tau})\right]\right\}\right\}
$$

where

$$
\vec{R}=\frac{1}{N} \int d \vec{r} \cdot \vec{r}|\psi(\vec{r}, \tau)|^{2}
$$

is the center of mass and $\vec{\rho}=\vec{\rho}-\vec{R}(\tau)$ is the coordinate in the new frame of reference. We obtain

$$
\begin{aligned}
& \ddot{\vec{R}}-4 \epsilon(\tau) \vec{R}=\overrightarrow{0} \\
& i \varphi_{\tau}+\nabla_{\rho^{\prime}}^{2} \varphi+|\varphi|^{2} \varphi+\epsilon(\tau) \rho^{\prime 2} \varphi=0 .
\end{aligned}
$$

Equations (3.3) and (3.4) show that in the parabolic potential, external (center of mass $\vec{R}$ ) and internal $[\varphi(\vec{\rho}, \tau)]$ 
degrees of freedom are separated. It is worth noting that such a separation takes place only in the case of the harmonic potential. Anharmonic terms in $U(\rho)$ lead to coupling between the external and internal degrees of freedom of the solitons. The soliton center of mass behaves like a time-dependent oscillator. To consider the internal motion of the soliton in the impurity field we introduce the change of variables called the lens transformation:

$$
\varphi(\vec{\rho}, \tau)=\frac{1}{b(\tau)} \Phi(\xi, T) \exp \left[i\left[T+\frac{\dot{b}}{4 b} \rho^{\prime 2}\right)\right] .
$$

Here

$$
\xi=\frac{\left|\rho^{\prime}\right|}{b(\tau)}
$$

is a new space variable, while

$$
T=\int_{0}^{\tau} d \widetilde{\tau} \frac{1}{b^{2}(\tau)}
$$

is a new time variable. As seen from Eqs. (3.5) and (3.6), the function $b(\tau)$ is the soliton width. Inserting Eq. (3.5) into Eq. (3.4) gives

$i \Phi_{T}+\left(\frac{\partial^{2}}{\partial \xi^{2}}+\frac{1}{\xi} \frac{\partial}{\partial \xi}\right) \Phi+|\Phi|^{2} \Phi-\Delta \xi^{2} \Phi-\Phi=0$,

where

$$
\Delta=\frac{1}{4} b^{3} \ddot{b}-\epsilon(\tau) b^{4} .
$$

Note that a transformation similar to Eq. (3.5) was used by Manassah [31] in his study of collapse in optical fibers with a parabolic profile of the index of refraction. But he investigated only the case which corresponds in our notation to $\Delta=0$ and $\epsilon(\tau)=$ constant. Karlsson, Anderson, and Desaix [32] also studied this problem applying the method of collective coordinates. We are interested in localized solutions $(\Phi \rightarrow 0$ for $\xi \rightarrow \infty)$ of Eq. (3.8). When $\Delta$ is a positive constant we can put $\Phi_{T}=0$ and Eq. (3.8) becomes

$$
\left[\frac{\partial^{2}}{\partial \xi^{2}}+\frac{1}{\xi} \frac{\partial}{\partial \xi}\right] \Phi-\Delta \xi^{2} \Phi+|\Phi|^{2} \Phi-\Phi=0 .
$$

For $\Delta>0$ Eq. (3.10) has localized solutions and in this case the wave function Eq. (3.5) describes a noncollapsing soliton. Exact solutions of Eq. (3.10) are unknown. We therefore apply a variational approach to obtain the dependence of the parameter $\Delta$ on the excitation number $N$. It is convenient to rescale variables

$$
\xi \rightarrow \Delta^{-1 / 4} \xi, \quad \Phi \rightarrow \Delta^{1 / 4} \Phi
$$

and we obtain instead of Eq. (3.10)

$$
\left(\frac{\partial^{2}}{\partial \xi^{2}}+\frac{1}{\xi} \frac{\partial}{\partial \xi}\right] \Phi+\left(|\Phi|^{2}-\xi^{2}\right) \Phi=\Delta^{-1 / 2} \Phi .
$$

Equation (3.12) is the Euler-Lagrange equation for the functional

$$
E=\int d \xi \xi\left\{\left|\frac{\partial \Phi}{\partial \xi}\right|^{2}-\frac{1}{2}|\Phi|^{4}+\xi^{2}|\Phi|^{2}+\Delta^{-1 / 2}|\Phi|^{2}\right\}
$$

As a trial function we use the function

$$
\Phi=\left(\frac{N}{2 \pi \ln 2}\right)^{1 / 2} \frac{1}{a} \operatorname{sech}\left(\frac{\xi}{a}\right),
$$

where $a$ is a trial parameter. Inserting Eq. (3.14) into Eq. (3.13) and minimizing with respect to $a$ we get

$$
a^{4}=\Delta=\frac{4}{27}\left(1-\frac{N}{N_{s}}\right) \frac{1+2 \ln 2}{\zeta(3)},
$$

where $\zeta$ denotes the Riemann zeta function and $N_{s}=4 \pi \ln 2(2 \ln 2+1) /(4 \ln 2-1) \simeq 11.7$ is the number of excitations in the ground state. Returning to the original variables, we obtain that at $N<N_{s}$ the soliton solution of Eq. (3.4) is represented by the function Eq. (3.5) with

$$
\Phi=\left(\frac{N}{2 \pi \ln 2}\right)^{1 / 2} \operatorname{sech} \xi
$$

The soliton dynamics in the parabolic potential Eq. (3.1) is governed by Eq. (3.3) for the center of mass and Eq. (3.9) for the width of the soliton. Equations (3.3) and (3.9) belong to the class of so-called Ermakov-Pinney equations [33,34] and, as was shown by Pinney [34], the solution to Eq. (3.9) can be written in the form

$$
b(t)=\left\{u^{2}(t)+\frac{4 \Delta}{W^{2}} v^{2}(t)\right\}^{1 / 2},
$$

where $W=\dot{u} v-u \dot{v}$ is the Wronskian and $u, v$ is the fundamental set of solutions of the respective linear equation

$$
\ddot{y}-4 \epsilon(t) y=0,
$$

which coincides with Eq. (3.3) for the center of mass motion.

\section{Special cases}

(i)

$$
\epsilon(\tau)=-\frac{\Omega_{0}^{2}}{4} \equiv-\frac{2|E| r_{0}^{2}}{z J l^{2}}=\text { const } .
$$

In this case the impurity is an acceptor of excitations and, as seen from Eq. (2.9), the quantity $|E|$ characterizes the depth of the well. Inserting Eq. (3.19) into Eqs. (3.3) and (3.17) yields in physical units

$$
\begin{aligned}
& R(t)=R_{0} \cos \omega\left(t+t_{0}\right), \\
& b^{2}(t)=B^{2} \cos ^{2} \omega\left(t+t_{1}\right)+\frac{z J l^{2} \Delta}{2|E| r_{0}^{2} B^{2}} \sin ^{2} \omega\left(t+t_{1}\right) .
\end{aligned}
$$

Here $R_{0}, B, t_{0}$ and $t_{1}$ are arbitrary constants,

$$
\omega=\frac{l}{r_{0}}\left[\frac{2 z|E| J}{\hbar^{2}}\right]^{1 / 2}
$$


is the frequency of the center of mass oscillations. The width $b(t)$ of the soliton oscillates with the double frequency. It is worth noting that the frequency $\omega$ depends on the depth $|E|$ of the impurity as well as the radius $r_{0}$ and on the matrix element $J$ of the excitation transfer. Note also that, as mentioned above, our approach is valid if the radius of the impurity $r_{0}$ exceeds the width of the soliton (i.e., $b<1$ ). Taking into account Eq. (3.21), we can arrive at the conclusion that the inequality

$$
\Delta<<\frac{|E| r_{0}^{2}}{2 z J l^{2}}
$$

must hold. Inserting Eq. (3.15) into inequality (3.23) shows that the condition Eq. (3.23) can be fulfilled in the easiest way when the impurity is not very deep $(|E|<<J)$ and when the number of excitations in the soliton is close to the threshold value $N_{s}$.

(ii)

$$
\epsilon(\tau)=-\frac{\Omega_{0}^{2}}{4}(1+\lambda \cos \Omega \tau) .
$$

This means that the acceptor molecule oscillates around its equilibrium position with the frequency $\Omega$. The molecule can perform either translational or librational motion. The parameter $\lambda$ is proportional to the amplitude of the impurity oscillations. Introducing Eq. (3.24) into Eq. (3.3) we get

$$
\ddot{R}+\Omega_{0}^{2}(1+\lambda \cos \Omega \tau) R=0,
$$

which is Mathieu's equation, and it is well known that the $\left(\Omega_{0}, \lambda\right)$ plane is divided into a region of stability [the function $R(\tau)$ is bounded as $\tau$ tends to infinity] and an instability region (parametric resonance). Let us consider the range of the first resonance

$$
\left|\Omega-2 \Omega_{0}\right|<\frac{\Omega_{0} \lambda}{2} .
$$

From Eq. (3.25) we get [35] the following approximate solution:

$R(\tau)=R_{0}\left\{e^{\gamma \tau} \cos \Omega\left(\tau+\tau_{0}\right)+e^{-\gamma \tau} \sin \Omega\left(\tau+\tau_{0}\right)\right\}$,

where $R_{0}$ and $\tau_{0}$ are constants that depend on the initial position of the soliton and its initial velocity. $\gamma$ is given as

$$
\gamma=\frac{1}{2}\left[\left(\frac{\Omega_{0} \lambda}{2}\right]^{2}-\left(\Omega-2 \Omega_{0}\right)^{2}\right]^{1 / 2} .
$$

It is seen from Eqs. (3.26)-(3.28) that when an acceptor molecule oscillates about its equilibrium position with a frequency in the range given by Eq. (3.26), it cannot trap the soliton. The amplitude of the oscillations of the soliton increases exponentially with the amplification coefficient $\gamma$ given by Eq. (3.28).

Using the split-step Fourier method [36], we have solved Eq. (2.10) numerically with $\epsilon(\tau)$ given according to Eqs. (3.19) and (3.24). Figure 1 shows in these two cases the behavior of the quantity

$$
V(t)=\int d \vec{r}|\psi(\vec{r}, t)|^{4} / \int d \vec{r}|\psi(\vec{r}, 0)|^{4},
$$



FIG. 1. Comparison of the numerical solution to Eq. (2.10) (solid lines) and the results of the analytical approach with $\epsilon(\tau)$ given by Eq. (3.19) (dashed line) and Eq. (3.24) (dotted line), respectively. The meaning of the depicted quantity is discussed in the text.

which characterizes the size of the excitation. In the framework of the self-similar solution, this quantity is given as

$$
V(t)=b_{0}^{2} b^{-2}(t)
$$

where $b_{0}=b(0)$. In Fig. 1 the dashed line is the result of the analytical approach with $\epsilon(\tau)$ constant [Eq. (3.19)] and the dotted line shows the result for $\epsilon(\tau)$ given by Eq.

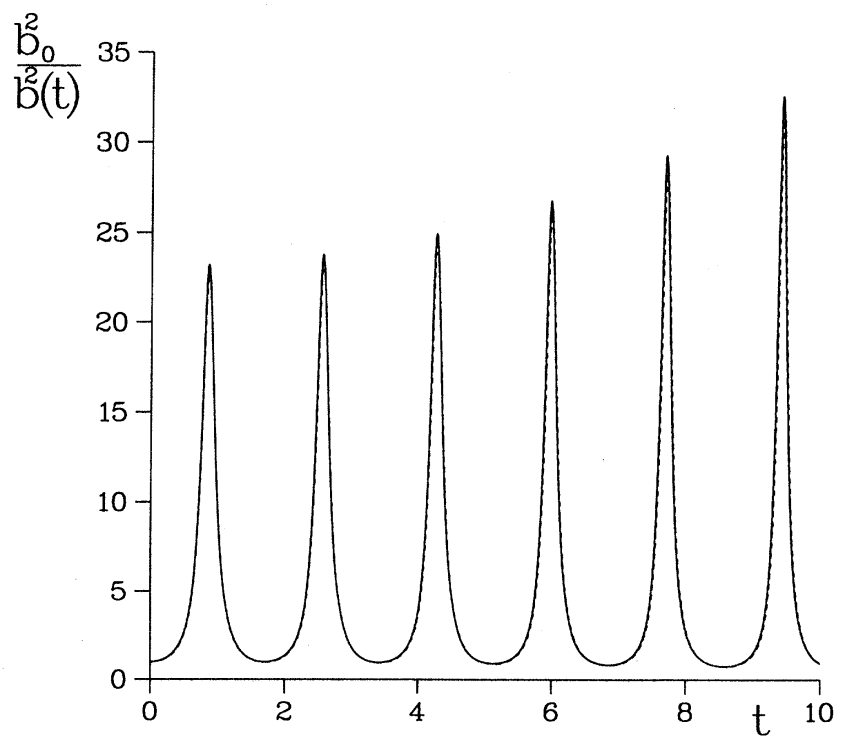

FIG. 2. Comparison of the numerical solution to Eq. (2.10) (solid lines) and the results of the analytical approach with $\epsilon(\tau)$ given by Eq. (3.24) (dashed line), where $\Omega_{0}=1.7, \Omega=2 \Omega_{0}$, and $\lambda=0.1$ are chosen such that the resonance effect develops fast. 


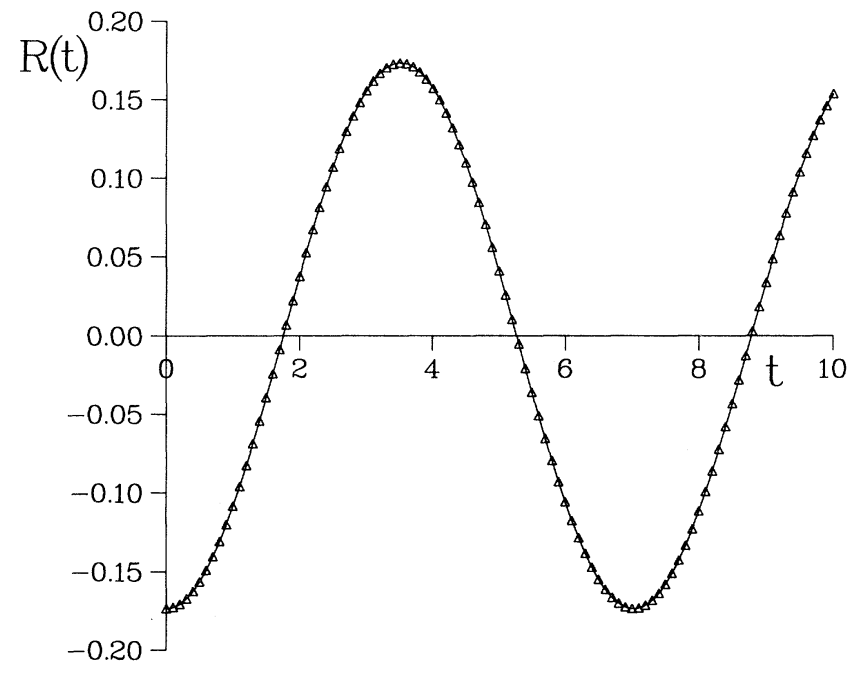

FIG. 3. The center of mass motion obtained from Eq. (2.10) (solid line) and the solution to Eq. (3.3) (indicated by triangles).

(3.24), while the solid lines indicate the results of the numerical solution of Eq. (2.10) with the above mentioned $\epsilon(\tau)$ dependences. It is seen that the analytical approach agrees quite well with the numerical simulations. Only near the maxima of the curves, where the excitations are very narrow, is there a small discrepancy.

In the case of $\epsilon(\tau)$ given by Eq. (3.24), the parameters (see figure captions) are chosen such that parametric resonance appears. The increasing amplitude indicates that this is the case. To investigate this further, we show in Fig. 2 a comparison of the numerical simulation (solid line) of Eq. (2.10) with $\epsilon(\tau)$ given by Eq. (3.24) and the analytical approach for parameters that gives fast development of the parametric resonance. It is clearly seen here that the resonance appears as predicted by the analysis.

Figure 3 shows (solid line) the motion of the center of mass in the numerical simulation of Eq. (2.10) with $\epsilon(\tau)$ given as in Eq. (3.24). The triangles indicate the analytical results. The agreement is in this case even better than in the case of $V(t)$, the reason being that the center of mass equation is derived without use of the self-similarity assumption.

\section{DIPOLELIKE EXCITATIONS}

As pointed out above, the soliton dynamics in the presence of an impurity is very complicated, in particular be- cause of a coupling between external and internal degrees of freedom of the soliton. We shall therefore assume that the center of mass of the soliton is immobile and that it coincides with the position of the impurity molecule $(\vec{R}(t)=\overrightarrow{0})$. It is our goal now to consider nonlinear stationary states of the two-dimensional system with an impurity which is an acceptor of excitation. Rose and Weinstein [37] proved that when the linear part of the Hamiltonian supports a bound state, the NLS equation has a stable nonlinear ground state when the excitation number is less than some critical value. In this section we are going to investigate the possibility of the creation of a dipolelike soliton state with an impurity. We shall treat the problem analytically, applying the method of collective coordinates. We introduce the test function

$$
\begin{aligned}
\psi(\vec{r}, t)=e^{i \mu t} & {\left[A_{1}(t) e^{i(\delta(t) / 2)} F\left(\left|\frac{\vec{r}-\vec{\rho}}{b}\right|\right)\right.} \\
& \left.-A_{2}(t) e^{-i[\delta(t) / 2]} F\left[\left|\frac{\vec{r}+\vec{\rho}}{b}\right|\right)\right],
\end{aligned}
$$

where $\mu$ is the nonlinear frequency, $\vec{\rho}$ is the position of the pulse, $b$ is its width $A_{n},(n=1,2)$ is the amplitude of the pulse, $\delta(t)$ is the phase difference, and $F(z)$ is the profile function. We shall use the Gaussian profile function

$$
F(x)=\exp \left(-\frac{x^{2}}{2}\right) .
$$

Such a choice of trial function $\psi(\vec{r}, t)$ signifies that we assume that the distance $\rho$ between pulses and their widths $b$ does not depend on time and we therefore restrict ourselves to the study of alternating shapes of the excitation. Inserting Eqs. (4.1) and (4.2) into the normalization condition Eq. (2.12) yields

$$
A^{2} \equiv A_{1}^{2}+A_{2}^{2}=\frac{N}{\pi b^{2}\left(1-\cos \xi \cos \delta e^{-\xi^{2}}\right)},
$$

where $\xi=\rho / b$, and the new variable

$$
\xi=-\frac{\pi}{2}+2 \tan ^{-1}\left(\frac{A_{1}}{A_{2}}\right)
$$

describes relative motion of the pulse maxima. Introducing Eq. (4.1), (4.2), and (4.3) into the Lagrange function

$L=\int d \vec{\rho}\left\{\operatorname{Im}\left(\psi^{*} \psi_{\tau}\right)+|\nabla \psi|^{2}+\frac{1}{2}|\psi|^{4}-U(\vec{\rho})|\psi|^{2}\right\}$

from which Eq. (2.10) can be derived, we obtain the effective Lagrangian

$$
\begin{aligned}
\frac{L}{N}=\mu+\frac{c}{2}\left(\dot{\delta} \sin \zeta-\dot{\zeta} \sin \delta e^{-\xi^{2}}\right)+\frac{c}{b^{2}}\left\{\left[1-\left(1-\xi^{2}\right) \cos \zeta \cos \delta e^{-\xi^{2}}\right]\right. & \left.-\frac{c N}{8 \pi}\left[2-\cos ^{2} \xi+\cos ^{2} \xi\left(1+2 \cos ^{2} \delta\right) e^{-2 \xi^{2}}-4 \cos \xi \cos \delta e^{-\left(3 \xi^{2} / 2\right)}\right]\right\} \\
- & \frac{|\epsilon| c}{\left(b^{2}+1\right)}\left(e^{-\xi^{2}\left(b^{2} / b^{2}+1\right)}-\cos \xi \cos \delta e^{-\xi^{2}}\right)
\end{aligned}
$$


where the abbreviation

$$
c=\frac{1}{1-\cos \xi \cos \delta e^{-\xi^{2}}}
$$

is used. It was also taken into account that the impurity is an acceptor of excitation $(\epsilon=-|\epsilon|)$. First of all, let us consider static solutions $\dot{\delta}=\dot{\xi}=0$. From Eq. (4.6) we see that there are two types of solutions:

$$
\xi=0, \delta=\pi
$$

and

$$
\xi=0, \delta=0 .
$$

It is seen from Eq. (4.1) that the first of them, Eq. (4.8), represents a symmetric state and at $\vec{\rho}=0$ it describes a radially symmetric bound state; this case was studied in [37]. We are here interested in dipolelike nonlinear excitations given by the second type of solution, Eq. (4.9). The stationary distance $\xi$ between pulses and their stationary width $b$ are the solutions to the equations

$$
\begin{aligned}
& \frac{\partial V(\xi, b)}{\partial \xi}=0, \\
& \frac{\partial V(\xi, b)}{\partial b}=0,
\end{aligned}
$$

where

$$
\begin{aligned}
V(\xi, b)=\{ & \left\{1-\left(1-\xi^{2}\right) e^{-\xi^{2}}\right. \\
& \left.-\frac{N}{8 \pi} \frac{1-4 e^{-\left(3 \xi^{2} / 2\right)}+3 e^{-2 \xi^{2}}}{1-e^{-\xi^{2}}}\right] \frac{1}{b^{2}} \\
& -\frac{|\epsilon|}{b^{2}+1}\left(e^{-\xi^{2}\left(b^{2} / b^{2}+1\right)}-e^{\left.-\xi^{2}\right)}\right\} \frac{1}{1-e^{-\xi^{2}}}
\end{aligned}
$$

is an effective potential function which is the Lagrange function Eq. (4.6) at $\delta=\xi=0$.

From Eqs. (4.11) and (4.12) we get

$$
\begin{aligned}
& \left(e^{\xi^{2}}-1\right)(1-n)+\xi^{2}-2 n \tanh \left[\frac{\xi^{2}}{4}\right]=|\epsilon| \frac{b^{4}}{\left(b^{2}+1\right)^{3}}\left[\left(\xi^{2}+b^{2}+1\right) \exp \left[\frac{\xi^{2}}{b^{2}+1}\right]-\left(b^{2}+1\right)\right] \\
& \left(1-e^{-\xi^{2}}\right)\left[1-\frac{n}{2} \operatorname{sech}^{2}\left[\frac{\xi^{2}}{4}\right]\right]-\xi^{2}+2 n \tanh \left[\frac{\xi^{2}}{4}\right]=|\epsilon| \frac{b^{2}}{\left(b^{2}+1\right)^{2}}\left[-\left(b^{2}+e^{-\xi^{2}}\right) \exp \left[\frac{\xi^{2}}{b^{2}+1}\right]+\left(b^{2}+1\right)\right]
\end{aligned}
$$

where the abbreviation $n=N /(8 \pi)$ was used. Eliminating $|\epsilon|$ from Eqs. (4.13) and (4.14), one can obtain an equation that connects $\xi, b$, and $n$. An analysis of this function $n(\xi, b)$ shows that it is a monotonous function of both variables $n \leq 1(N \leq 8 \pi)$. It is worth noting that in the framework of the Gaussian test function Eq. (4.2) the collapse in the nonimpurity case occurs when the number of excitations exceeds $4 \pi$. Thus the inequality $N \leq 8 \pi$ means that without the impurity the pulse will disperse. To consider the possibility of collapse, one has to modify the function Eq. (4.1) taking into consideration the time dependence of the width $b$. This we will consider in a future paper. Here we restrict ourselves to the case where the pulses cannot collapse.

When the distance between pulses is small $(\xi \leq 1)$, one can expand Eqs. (4.13) and (4.14) in powers of $\xi^{2}$ and get

$$
\begin{aligned}
& 1-\frac{3}{4} n+\frac{1}{4}(1-n) \xi^{2}=\frac{|\epsilon| b^{4}}{\left(b^{2}+1\right)^{3}}\left[1+\frac{3}{4} \frac{\xi^{2}}{b^{2}+1}\right] \\
& 1-\frac{n}{2}-\frac{|\epsilon| b^{4}}{\left(b^{2}+1\right)^{3}}=\frac{1}{3}\left[1-\frac{3}{8} n-\frac{|\epsilon| b^{4}\left(b^{2}-1\right)}{\left(b^{2}+1\right)^{4}}\right] \xi^{2}
\end{aligned}
$$

Omitting in Eq. (4.15) small terms proportional to $\xi^{2}$, we obtain that Eq. (4.15) has solutions when

$$
|\epsilon|>\epsilon_{t} \equiv \frac{27}{4}\left[1-\frac{3}{4} n\right]
$$

and from Eq. (4.16) we obtain an approximate expression for the equilibrium distance between the pulses

$$
\xi=\left(\frac{3 n}{4}\right]^{1 / 2}\left[\frac{3 n}{8}+2\left[1-\frac{3 n}{4}\right]\left(b^{2}+1\right)^{-1}\right]^{-1 / 2} \text {. }
$$

It is seen from inequality equation (4.17) that the dipolelike bound state can exist if the strength of the impurity exceeds some critical value $\epsilon_{t}$ which depends on the excitation number $N$. From Eq. (4.15) we see that the equilibrium width $b$ monotonically decreases when the impurity strength increases. At large $|\epsilon|$ we have

$$
b \simeq|\epsilon|^{-1 / 4}\left(1-\frac{3 n}{4}\right)^{-1 / 4} \text {. }
$$

The distance between pulses Eq. (4.18) increases when the excitation number increases but for $N$ close to $8 \pi$ we cannot use Eqs. (4.15) and (4.16). To obtain the solution of Eqs. (4.13) and (4.14) in a wider range of excitation numbers, we assume that $|\epsilon| \gg \epsilon_{t}$. In this case the widths of the pulses are small and one can expand Eqs. (4.13) and (4.14) in powers of $b^{2}$. Taking into account only leading terms of the expansion, we get

$$
|\epsilon| b^{4}=\frac{\left(e^{\xi^{2}}-1\right)(1-n)+\xi^{2}-2 n \tanh \left(\xi^{2} / 4\right)}{\left(\xi^{2}+1\right) e^{\xi^{2}-1}},
$$

and the equilibrium distance $\xi$ is given by the implicit function 


$$
\begin{aligned}
& \left\{\left(e^{\xi^{2}}-1\right)^{2}-\xi^{2}\left(e^{\xi^{2}}-1\right)+2\left[\xi^{2} e^{\xi^{2} / 2}+2\left(1-\xi^{2} e^{-\xi^{2} / 2}\right) \sinh \left[\frac{\xi^{2}}{2}\right]\right] \tanh \left[\frac{\xi^{2}}{4}\right]\right\} n \\
& =\left(e^{\xi^{2}}-1\right)^{2}-\xi^{4}\left(e^{\xi^{2}+1}\right)+4 \sinh ^{2}\left[\frac{\xi^{2}}{2}\right] \text {. }
\end{aligned}
$$

Note that in this case $\left(|\epsilon| \gg \epsilon_{t}\right)$ the equilibrium distance between pulses does not depend on the impurity strength. The dependences of the pulse width as well as of the distances between pulses that follow from Eqs. (4.20) and (4.21) are shown in Fig. 4. The widths of the pulses become equal to zero when $N \rightarrow 8 \pi$. The distance between the pulses $\rho=\xi b$ is a nonmonotonic function of $N$; when $N \rightarrow 0$ it tends to zero according to Eq. (4.18). When $N \rightarrow 8 \pi, \xi$ is increased as $\sqrt{-\ln (1-n)}$ but the widths of the pulses decrease more rapidly.

We have checked numerically the existence of a threshold value of the impurity strength for the creation of a dipolelike bound state. The results are shown in Fig. 5. It is seen that the quantity $V(t)$ [see Eq. (3.29)] that characterizes the size of the excitations tends to zero at small $|\epsilon|$ (it means that the excitation disperses). When $|\epsilon|$ exceeds some critical value [in the case $N \geq N_{s}$ this critical value is around 4.5, which is also the order of magnitude predicted by Eq. (4.16)] $V(t)$ becomes finite after some transient period.
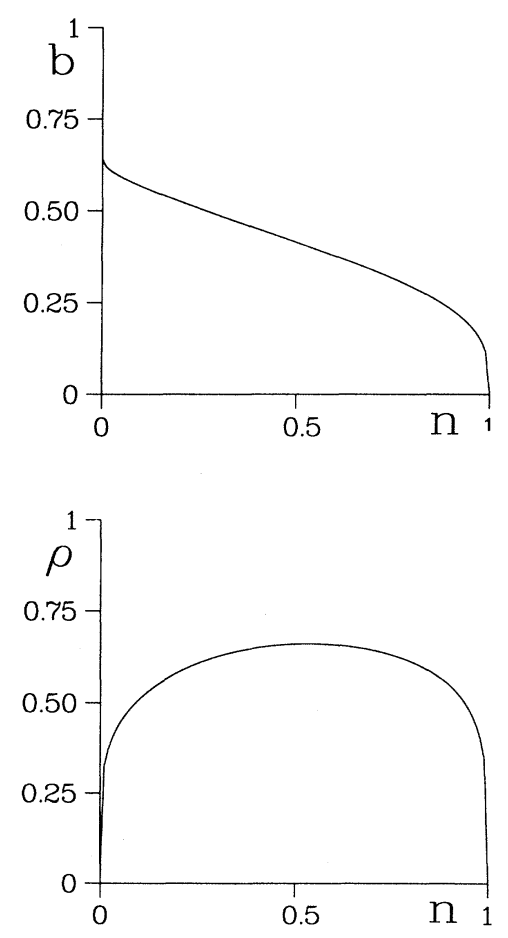

FIG. 4. The pulse width $b$, according to Eq. (4.20), of the stationary dipolelike excitation as a function of the normalized number $n$ (upper part). The distance $\rho=\xi b$ between pulses as obtained from Eq. (4.21) of the stationary dipolelike excitations as a function of the normalized number $n$ (lower part ).
Based on the results of the numerical simulations, we calculated the mean-square deviations

$$
\left\langle(\Delta V)^{2}\right\rangle=\left\langle V^{2}\right\rangle-\langle V\rangle^{2} \text {. }
$$

Here

$$
\langle f\rangle=\frac{1}{T} \int_{t_{0}}^{T+t_{0}} d \widetilde{t} f(\widetilde{t})
$$

is the mean value of the function $f(t)$ on the interval $\left[t_{0}, t_{0}+T\right] ; t_{0}$ was chosen equal to 1.0 and $T=30$. The result of this is that $|\epsilon|>\epsilon_{\mathrm{cr}}\left\langle(\Delta V)^{2}\right\rangle=3 \times 10^{-3}$. We can then draw the conclusion that in this case the dipolelike nonlinear excitation exists as a stationary state.

Figure 6 depicts the distribution of the real part and imaginary part of the wave function $\psi(\vec{r}, t)$ for $|\epsilon|=5.0$, $N=11.7$, and $t=30$. Let us now consider dynamical properties of the dipolelike excitation. We shall consider small oscillations and expand the Lagrangian Eq. (4.6) taking into account only small deviations from the equilibrium position given by Eq. (4.9). In this case we obtain from the Lagrangian Eq. (4.6)

$$
L=N\left\{\frac{1}{2}\left(\dot{\delta} \xi-\dot{\zeta} \delta e^{-\xi^{2}}\right)-B_{\xi} \frac{\xi^{2}}{2}-B_{\delta} \frac{\delta^{2}}{2}\right\}
$$

Here



FIG. 5. The inverse width squared obtained by numerical solution of Eq. (2.10). $N=11.7$ and from the bottom we have $\epsilon=4.0,4.2,4.4,4.5,4.6,5.0$. The dashed line indicates the average of the curve for $\epsilon=5.0$. 


$$
\begin{aligned}
& B_{\zeta}=\frac{1}{b^{2}\left(1-e^{\left.-\xi^{2}\right)^{2}}\right.}\left[\xi^{2} e^{-\xi^{2}}+\frac{2 n}{1-e^{-\xi^{2}}}\left(1-2 e^{-\xi^{2}}-2 e^{-(5 / 2) \xi^{2}}-3 e^{-2 \xi^{2}}+2 e^{-(3 / 2) \xi^{2}}\right)\right. \\
&+\frac{|\epsilon| b^{2}}{b^{2}+1} e^{\left.-\xi^{2}\left(1-e^{-\left[b^{2} /\left(b^{2}+1\right)\right] \xi^{2}}\right)\right]} \\
& B_{\delta}=\frac{1}{b^{2}\left(1-e^{\left.-\xi^{2}\right)^{2}}\right.}\left[\xi^{2} e^{-\xi^{2}}+\frac{2 n}{1-e^{-\xi^{2}}}\left(-e^{-\xi^{2}}+2 e^{-(5 / 2) \xi^{2}}-2 e^{-2 \xi^{2}}+2 e^{-(3 / 2) \xi^{2}}-e^{-3 \xi^{2}}\right)\right. \\
& \\
&+\frac{|\epsilon| b^{2}}{b^{2}+1} e^{\left.-\xi^{2}\left(1-e^{-\left[b^{2} /\left(b^{2}+1\right)\right] \xi^{2}}\right)\right]}
\end{aligned}
$$

are the elastic coefficients. The equilibrium distance $\xi$ and width are given by Eqs. (4.17) and (4.18). It is seen that both coefficients are positive for the excitation number $N$ under consideration.

From the Lagrangian Eq. (4.24) we get that the equation of motion for the phase difference $\delta(t)$ has the form

$$
\ddot{\delta}+\delta \frac{4 B_{\zeta} B_{\delta}}{\left(1+e^{-\xi^{2}}\right)^{2}}=0
$$

and consequently

$$
\delta(t)=\delta_{0} \cos \frac{2 \sqrt{B_{\zeta} B_{\delta}}}{1+e^{-\xi^{2}}} t .
$$

It means that the components of the dipolelike excitation take part in two types of oscillations. The first one occurs with the nonlinear frequency $\mu$ whose value depends on the energy of the system. The relative motions of the pulse maxima are described in Eq. (4.28). It is seen that the period of these oscillations depends on the excitation number $N$, impurity strength $|\epsilon|$. For example, for $|\epsilon| \simeq 5$ and $N \simeq N_{s}$ the period of the relative oscillations is of the order of 1 . This estimation is in agreement with the results of the numerical simulations, which are presented in Fig. 7.

Thus, one can conclude that as a result of the interaction between the nonlinear excitations and impurity, a di-
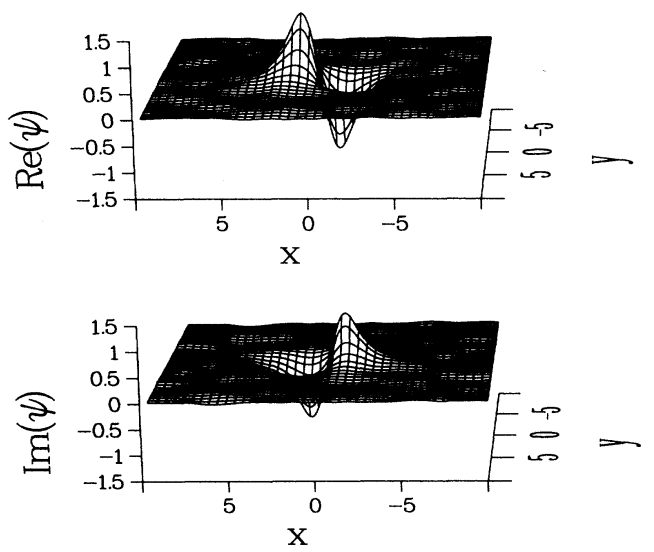

FIG. 6. The real and imaginary parts of the stationary state at $N=11.7$ for $\epsilon=5.0$ at $t=40$. polelike nonlinear bound state can exist. The stability of this state and its dynamical properties depend on the relation between the strength of the impurity $|\epsilon|$ and the excitation number $N$.

\section{ESTIMATION OF MODEL PARAMETERS}

There are two dimensionless parameters that determine the behavior of solitons in the presence of an impurity: the number of excitations $N$ and the power of the impurity $\epsilon$. The parameters $N$ characterizes the nonlinear properties of the system. As it follows from Eq. (2.12), (A18), and (A19), it can be written in the following form:

$$
N=\frac{\alpha^{2}}{2} \frac{z D^{2}}{M c^{2} J}
$$

Here $D=\left.D_{\vec{n}, \vec{n}}\right|_{\vec{n}-\vec{n}=\vec{\delta}}$, where $D_{\vec{n}, \vec{n}}$ is the change in

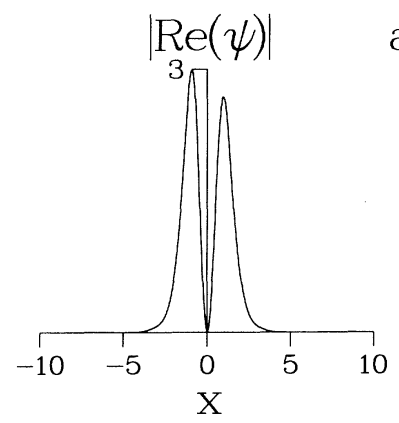

a)
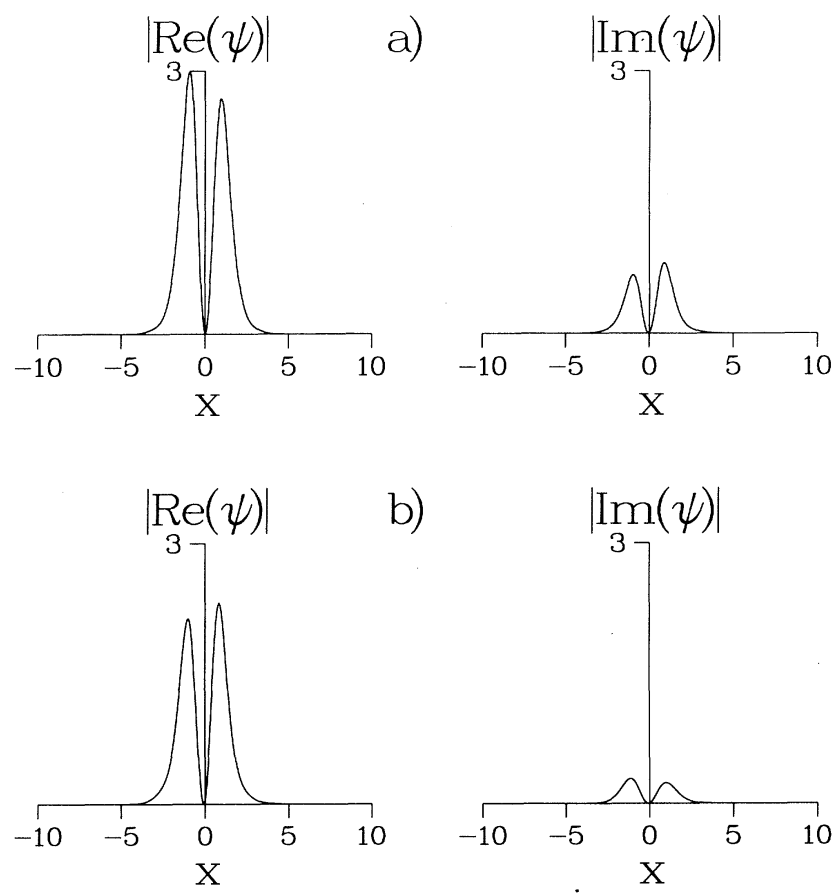

b)

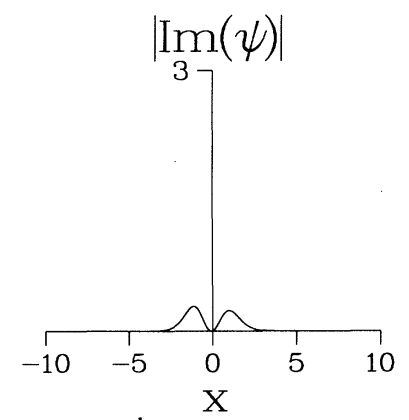

FIG. 7. Illustrating the relative oscillations, we show the absolute value of the real part and the imaginary part at time $t=30$ (a) and $t=31$ (b). The parameters are $N=11.7$ and $\epsilon=5.0$. 
the interaction energy between the molecules $\vec{n}$ and $\vec{n}^{\prime}$ in the transition of one of them to the excited state, the parameter $\alpha$ characterizes the dependence of this matrix element on distance between molecules

$$
\alpha=\frac{d \ln D_{\vec{n}, \overrightarrow{0}}}{d \vec{n}} .
$$

In accordance with the theory of molecular excitations [1], the quantity $z D \equiv \sum_{\vec{n}} D_{\vec{n}, 0}$ is the change of excitation energy of a molecule in a crystal with respect to the gas phase. One can also say that the quantity $D$ is approximately equal to the difference

$$
D=\left|\Delta E^{\prime}-\Delta E\right| \text {, }
$$

where $\Delta E$ is the excitation energy of a dye monomer and $\Delta E^{\prime}$ is the excitation energy of a dye dimer. We estimate the values of the parameters appearing in our theory for the oxacyanine Scheibe aggregates studied by Czikkely, Fösterling, and Kuhn [11] and Möbius and Kuhn [14,15]. It was shown in Ref. [11] that the typical value of the monomer to dimer band shift is $0.2 \mathrm{eV}$. It means that

$$
D \simeq 3 \times 10^{-20} \mathrm{~J} .
$$

The remaining parameters entering Eq. (5.1) were estimated in Refs. [14,15,23] from the monolayer oxacyanine Scheibe aggregates:

$M=1.2 \times 10^{-24} \mathrm{~kg}, \quad J=3.6 \times 10^{-21} \mathrm{~J}, \quad c=10^{3} \mathrm{~m} / \mathrm{s}$.

Inserting Eqs. (5.3) and (5.4) into Eq. (5.1) yields

$$
N=0.1 \alpha^{2} z
$$

Thus if we assume that the interaction $D_{\vec{n}, \vec{n}}$, has a van der Waals character $(\alpha=6)$ and the number of nearest neighbors is $z=4$, we get

$$
N=14.4 \text {, }
$$

which exceeds the threshold number $N_{s}=11.7$. It is worth noting that according to Eq. (5.1) the number of excitations $N$ is proportional to the square of the gas to crystal absorption band shift $D$. Thus one can expect manifestations of nonlinear effects in such molecular system where this shift is significant.

Another dimensionless parameter that determines the soliton behavior is the power of the impurity $\epsilon$. As seen from Eq. (2.11), it depends on both the strength of the impurity $E$ and its radius $r_{0}$. The impurity strength can roughly be estimated as the distance between the maximum of sensitized (acceptor) fluorescence and the maximum of the host fluorescence. Using the data of Möbius and Kuhn [14,15], one can obtain that, for instance, in the case of a monolayer oxacyanine dye Scheibe aggregate doped with a thiacyanine dye,

$$
E \simeq 0.36 \mathrm{eV}=6 \times 10^{-20} \mathrm{~J} .
$$

Thus, from Eqs. (2.11) and (5.5) we get

$$
\epsilon=\frac{32}{z}\left(\frac{r_{0}}{l}\right)^{2}=8\left[\frac{r_{0}}{l}\right)^{2},
$$

where the assumption $z=4$ was used. As regards the radius of the impurity, we cannot estimate it using available experimental data. It is interesting to note, however, that in the case of an oxacyanine dye monolayer where the number of excitations is given by Eq. (5.7), the condition for the existence of dipolelike bound states Eq. (4.17) reads

$$
r_{0}^{2}>0.45 l^{2} \text {. }
$$

Thus one can expect that even relatively short range impurities can bound a nonlinear excitation and create a dipolelike stationary state.

\section{SUMMARY AND CONCLUSIONS}

In this paper, we developed a theoretical framework for investigating nonlinear dynamical properties of electronic excitations in two-dimensional molecular structures with impurities. The equation governing the dynamics of the excitations is the two-dimensional NLS equation with a variable coefficient that represents the influence of the impurity molecule.

Transforming the NLS equation to a noninertial frame of reference coupled with the center of mass of the soliton, we investigate the soliton motion in the close vicinity of an impurity when the impurity potential can be considered as parabolic. Using the so-called lens transformation, we show that the problem under consideration may be reduced to Ermakov-Pinney equations. Comparison between the results that were obtained by solving the ordinary differential equations (Ermakov-Pinney equations) and the original NLS equation show that the approaches are in good agreement.

We study bound states of the solitons with the impurity and show that when the number of excitations in the soliton $N$ is less than $2 N_{s}\left(N_{s}\right.$ is the number of excitations in the ground state soliton), a dipolelike soliton appears. It exists even at $N>N_{s}$ when the symmetric bound state collapses. We also consider dynamical properties of the dipolelike bound state and derive an effective Lagrangian that describes the alternation of the shape of the solitons. Analytical results are in qualitative agreement with the results of the numerical simulations.

The theory contains two dimensionless parameters: the number of excitations and the power of the impurity $\epsilon$. We estimate them for oxacyanine Scheibe aggregates studied by Möbius and Kuhn [14,15] and by Czikkely, Fösterling, and Kuhn [11] and show that in a previous paper [23] we underestimated the role of the interaction of excitons with acoustic phonons. But it may be important particularly in molecular systems with a large gas to crystal absorption band shift (the $D$-term in the theory of molecular excitons [1]) and rapidly decaying intermolecular interactions. Our estimations show that in the case of oxacyanine Scheibe aggregates, the nonlinearity parameter caused by the exciton-acoustic-phonon interaction is 
large enough for the nonlinear properties of the electronic excitations to become significant.

Estimation of the impurity power cannot be done because of lack of experimental data, but preliminary results show that, for instance, the creation of the dipolelike bound state of a soliton with the impurity is likely.

\section{ACKNOWLEDGMENTS}

We wish to thank $H$. Kuhn for providing detailed information about the thiacyanine molecules in the Scheibe aggregate. J. B. Keller, J. Juul Rasmussen, and L. Bergé are thanked for fruitful discussions. Yu. G. would like to express his thanks for the hospitality of the MIDIT center at the Technical University of Denmark (DTU) where this work was done.

\section{APPENDIX}

In our recent paper [23], the effective nonlinear Hamiltonian Eq. (2.1) was derived taking into account interaction of the electronic excitations with optical phonons (it was assumed that the displacements under consideration are characterized by a finite frequency $\omega_{0}$ and in this way they correspond to rotational molecular vibrations). In this Appendix, we want to show that the interaction with acoustic phonons in planar molecular systems may contribute significantly to the nonlinear properties of the electronic excitations. For the sake of simplicity we shall consider a nonimpurity case. According to the theory of molecular excitons [1] the exciton-phonon Hamiltonian of the molecular system can be written as follows:

$$
\begin{aligned}
H= & \sum_{\vec{n}, \vec{n}}\left(E_{n} \delta_{\vec{n}, \vec{n}}-J_{\vec{n}, \vec{n}^{\prime}}\right) B_{\vec{n}}^{\dagger} B_{\vec{n}}+\sum_{\vec{q}, s} \hbar \omega_{s}(\vec{q}) b_{\vec{q}, s}^{\dagger} b_{\vec{q}, s} \\
& +\frac{1}{\sqrt{N_{0}}} \sum_{\vec{q}, s} \sum_{\vec{n}} \chi_{\vec{q}, s} e^{i \vec{q} \cdot \vec{n}} B_{\vec{n}}^{\dagger} B_{\vec{n}}\left(b_{\vec{q}, s}+b_{-\vec{q}, s}^{\dagger}\right)
\end{aligned}
$$

Here $B_{\vec{n}}^{\dagger}\left(B_{\vec{n}}\right)$ is the creation (annihilation) operator of the electronic excitations on the molecular $\vec{n}, b_{\vec{q}, s}^{\dagger}\left(b_{\vec{q}, s}\right)$ is the creation (annihilation) operator for the acoustic phonon of the branch $s$ with the wave vector $\vec{q}$ and the frequency $\omega_{s}(\vec{q}) . N_{0}$ is the number of molecules in the system. The exciton-phonon coupling function has the form

$\chi_{\vec{q}, s}=\sum_{\vec{n}}\left[\frac{\hbar}{2 M \omega_{s}(\vec{q})}\right]^{1 / 2}\left(1-e^{\vec{q} \cdot \vec{n}}\right) \overrightarrow{e_{s}}(\vec{q}) \cdot \frac{\partial}{\partial \vec{n}} D_{\vec{n}, 0}$,

where $D_{\vec{n} \vec{n}}$ is the change in the interaction energy of the $\vec{n}$ th molecule with the $\vec{n}^{\prime}$ th molecule in the transition of one of these molecules to the electronic excited state, $\overrightarrow{e_{s}}(\vec{q})$ is the unit polarization vector, $M$ is the mass of a molecule. It is convenient to use the same approach as in Ref. [23]. Introducing functions

$$
\begin{aligned}
& \rho_{\vec{n}, \vec{n}}(t)=\operatorname{Tr}\left\{\rho(t) B_{\vec{n}}^{\dagger} B_{\vec{n}}\right\}, \\
& \beta_{\vec{q}, s}(t)=\operatorname{Tr}\left\{\rho(t) b_{\vec{q}, s}\right\},
\end{aligned}
$$

where $\rho(t)$ is a nonequilibrium density matrix and $\operatorname{Tr}$ denotes the trace, we obtain from the Hamiltonian Eq. (A1) that

$$
\begin{aligned}
i \hbar \dot{\rho}_{\vec{n}, \vec{n}}= & \sum_{\vec{e}}\left(J_{\vec{n}, \vec{e}} \rho_{\vec{e}, \vec{n}}-J_{\vec{n}, \vec{e}} \rho_{\vec{n}, \vec{e}}\right) \\
& +\frac{1}{\sqrt{N_{0}}} \sum_{\vec{q}, s} \chi_{\vec{q}, s}\left(\beta_{\vec{q}, s}+\beta_{-\vec{q}, s}^{*}\right) \\
& \times\left(e^{i \vec{q} \cdot \vec{n}}-e^{i \vec{q} \cdot \vec{n}}\right) \rho_{\vec{n}, \vec{n}}, \\
i \hbar \dot{\beta}_{\vec{q}, s}= & \hbar \omega_{s}(\vec{q}) \beta_{\vec{q}, s}+\frac{1}{\sqrt{N_{0}}} \sum_{\vec{n}} \chi_{\vec{q}, s}^{*} e^{-i \vec{q} \cdot \vec{n}} \rho_{\vec{n}, \vec{n}},
\end{aligned}
$$

where the decoupling

$\operatorname{Tr}\left\{\rho(t) B_{\vec{n}}^{\dagger} B_{\vec{n}}\left(b_{\vec{q}, s}+b_{-\vec{q}, s}^{\dagger}\right)\right\} \simeq \rho_{\vec{n}, \vec{n}^{\prime}}(t)\left(\beta_{\vec{q}, s}+\beta_{-\vec{q}, s}^{*}\right)$

was used. Considering the steady state solution to Eq. (A6). $\left(\dot{\beta}_{\vec{q}, s}=0\right)$, we get

$$
\beta_{\vec{q}, s}=-\frac{1}{\sqrt{N_{0}}} \sum_{\vec{n}} \frac{\chi_{\vec{q}, s}^{*} e^{-\vec{q} \cdot \vec{n}}}{\hbar \omega_{s}(\vec{q})} \rho_{\vec{n}, \vec{n}} .
$$

From Eqs. (A5) and (A8), it follows that $\rho_{\vec{n}, \vec{n}}$ may be written as a product

$$
\rho_{\vec{n}, \vec{n}}=\phi_{\vec{n}}^{*}(t) \phi_{\vec{n}}(t),
$$

where the wave function $\phi_{\vec{n}}(t)$ satisfies the equation

$$
i \hbar \dot{\phi}_{\vec{n}}+\sum_{\vec{n}} J_{\vec{n}, \vec{n}^{\prime}} \phi_{\overrightarrow{n^{\prime}}}+\sum_{\overrightarrow{n^{\prime}}} V_{\vec{n}, \vec{n}^{\prime}}\left|\phi_{\vec{n}}\right|^{2} \phi_{\overrightarrow{n^{\prime}}}=0,
$$

where the nonlinearity matrix element

$$
V_{\vec{n}, \vec{n}}=\frac{1}{N_{0}} \sum_{\vec{q}, s} \frac{2\left|\chi_{\vec{q}, s}\right|^{2}}{\hbar \omega_{s}(\vec{q})} e^{i \vec{q} \cdot(\vec{n}-\vec{n})}
$$

Let us now estimate the nonlinearity matrix element $V_{\vec{n}, \vec{n}}$, for the case where the matrix element of the intermolecular interaction $D_{\vec{n}, \vec{n}}$ depends only on the distance between molecules and can be modeled in the form

$$
D_{\vec{n}, \vec{n}}=D\left(\frac{l}{\left|\vec{n}-\vec{n}^{\prime}\right|}\right)^{\alpha},
$$

where $D$ and $\alpha$ are the parameters of the interaction. Inserting Eq. (A12) into Eq. (A2) yields

$$
\chi_{\vec{q}, s}=-\alpha\left(\frac{\hbar}{2 M \omega_{s}(\vec{q})}\right)^{1 / 2} \sum_{\vec{n}}\left(1-e^{\vec{q} \cdot \vec{n}}\right) \frac{\vec{e}_{s}(\vec{q}) \vec{n}}{n^{2}} D_{\vec{n}, \overrightarrow{0}}
$$

In the following we shall assume that the interaction $D_{\vec{n}, \vec{n}}$ is short-range (e.g., $\alpha=6$ for the van der Waals interaction) and we can take into account in Eq. (A13) molecules in the first coordination sphere. We shall assume also that our lattice may be approximated by an isotopic elastic system. This means that we consider lattices where the number of atoms in the first coordination sphere is large enough (e.g., in the trigonal lattice the number of the nearest neighbors $z$ is 6 but together with the next ones it is 12) and the relation

$$
\frac{1}{z} \underset{\vec{\delta}}{ }=\frac{1}{2 \pi} \int_{0}^{2 \pi} d \theta
$$


will be used, with $\vec{\delta}$ being the vector that connects a molecule with molecules of its first coordination sphere (in 2D) of the radius $l$ :

$$
\vec{\delta}=(l \cos \theta, l \sin \theta) .
$$

Using Eqs. (A14) and (A15), we obtain that for small $q$ the exciton-phonon coupling function Eq. (A13) can be written as follows:

$$
\begin{aligned}
\chi_{\vec{q}, s} & =i \alpha D\left[\frac{\hbar}{2 M \omega_{s}(\vec{q})}\right]^{1 / 2} \frac{1}{l^{2}} \sum_{\vec{\delta}}(\vec{q} \cdot \vec{\delta})\left[\vec{e}_{s}(\vec{q}) \cdot \vec{\delta}\right] \\
& =i \frac{1}{2} z \alpha D\left[\frac{\hbar}{2 M \omega_{s}(\vec{q})}\right]^{1 / 2}\left[\vec{e}_{s}(\vec{q}) \cdot \vec{q}\right]
\end{aligned}
$$

It is seen from Eq. (A16) that in this approximation only longitudinal acoustic phonons $(s=\|)$ with $\vec{e}_{s}(\vec{q}) \| \vec{q}$ contribute to the exciton-phonon coupling function, Eq. (A2), and to the nonlinearity matrix element $V_{\vec{n}, \vec{n}}$ given by Eq. (A11). Transversal phonons can contribute to the nonlinear matrix element only in anisotropic lattices. Inserting Eq. (A16) into Eq. (A11) and taking into account that in the long wave limit the frequency of the longitudinal acoustic phonons can be represented in the form

$$
\omega_{\|}(q)=c q
$$

where $c$ is the speed of the longitudinal sound in the lattice, we obtain that the nonlinearity matrix element caused by the interaction of excitons with acoustic phonons as given by

$$
V_{\vec{n}, \vec{n}^{\prime}}=V_{a} \delta_{\vec{n}, \vec{n}^{\prime}},
$$

where

$$
V_{a}=\frac{\alpha^{2}}{4} \frac{(z D)^{2}}{M c^{2}}
$$

is the nonlinearity parameter. Thus, from Eqs. (A10) and (A18) we draw the conclusion that the effective Hamiltonian for electronic excitations has the form

$H=\sum_{\vec{n}}\left\{E_{\vec{n}}\left|\phi_{\vec{n}}\right|^{2}-\frac{1}{2} V\left|\phi_{\vec{n}}\right|^{4}-\sum_{\vec{n}} J_{\vec{n}, \vec{n}} \phi_{\vec{n}}^{*} \phi_{\vec{n}}\right\}$.
[1] A. S. Davydov, Theory of Molecular Excitons (Plenum, New York, 1971).

[2] V. M. Agranovich and M. D. Galanin, Electronic Excitations Energy Transfer in Condensed Matter (NorthHolland, Amsterdam, 1988).

[3] R. S. Knox in Primary Processes of Photosynthesis, edited by J. Barber (North-Holland, Amsterdam, 1977).

[4] A. S. Davydov, Biology and Quantum Mechanics (Pergamon, Oxford, 1982).

[5] G. Feher and M. Y. Okamura, in The Photosynthetic Bacteria, edited by R. Clayton and W. Sistrom (Plenum, New York, 1978).

[6] P. B. Gilman, Photogr. Sci. Eng. 18, 418 (1978).

[7] Y. Wang, Chem. Phys. Lett. 126, 209 (1986).

[8] H. Kuhn, D. Möbius, and H. Bucher, in Physical Methods of Chemistry, edited by A. Weissberger and B. Rossiter (Wiley, New York, 1972), Vol 1B.

[9] L. M. Blinov, Russ. Chem. Rev. 52, 713 (1983).

[10] R. H. Tredgold, Order in Thin Organic Films (Cambridge University Press, Cambridge, England, 1994).

[11] V. Czikkely, H. D. Fösterling, and H. Kuhn, Chem. Phys. Lett. 6, 11 (1970).

[12] G. Scheibe, Angew. Chem. 50, 212 (1937).

[13] E. E. Jelly, Nature 10, 631 (1937).

[14] D. Möbius and H. Kuhn, Isr. J. Chem. 18, 375 (1979).

[15] D. Möbius and H. Kuhn, J. Appl. Phys. 64, 5138 (1988).

[16] S. De Boer and D. A. Wiersma, Chem. Phys. Lett. 165, 45 (1990).

[17] F. C. Spano and S. Mukamel, J. Chem. Phys. 91, 683 (1989).

[18] E. A. Bartnik and K. J. Blinowska, Phys. Lett. A 134, 448 (1989); E. A. Bartnik, K. J. Blinowska, and J. A. Tuszyński, Phys. Lett. A 159, 67 (1991); E. A. Bartnik and
J. A. Tuszyński, Phys. Rev. E 48, 1516 (1993).

[19] G. C. Huth, F. Gutmann, and G. Vitiello, Phys. Lett. A 154, 381 (1991).

[20] P. L. Christiansen, S. Pagano, and G. Vitiello, Phys. Lett. A 154, 498 (1991).

[21] P. S. Lomdahl, O. H. Olsen, and P. L. Christiansen, Phys. Lett. A 78, 125 (1980).

[22] J. Juul Rasmussen and K. Rypdal, Phys. Scr. 33, 481 (1986).

[23] O. Bang, P. L. Christiansen, F. If, K. Ø. Rasmussen, and Y. B. Gaididei, Phys. Rev. E 49, 4627 (1994).

[24] O. Bang, P. L. Christiansen, F. If, K. Ø. Rasmussen, and Yu. B. Gaididei, Appl. Anal. 57, 3 (1995).

[25] K. Ø. Rasmussen, Yu. B. Gaididei, O. Bang, and P. L. Christiansen, Physica D (to be published).

[26] Y. S. Kivshar and B. Malomed, Rev. Mod. Phys. 61, 763 (1989).

[27] R. Scharf and A. R. Bishop, Phys. Rev. A 43, 6535 (1991).

[28] D. Anderson, M. Bonnedal, and M. Lisak, Phys. Fluids 22, 1838 (1979).

[29] H. H. Chen and C. S. Liu, Phys. Fluids 21, 377 (1978).

[30] Y. Nogami and F. M. Toyama, Phys. Rev. E 49, 4497 (1994).

[31] J. T. Manassah, Opt. Lett. 17, 1259 (1992).

[32] M. Karlsson, D. Anderson, and M. Desaix, J. Opt. Soc. Am. 17, 22 (1992).

[33] V. P. Ermakov, Univ. Izv. Kiev 20, 1 (1880).

[34] E. Pinney, Proc. Amer. Math. Soc. 1, 681 (1950).

[35] L. D. Landau and E. M. Lifshitz, Mechanics (Pergamon, Oxford, 1960).

[36] R. T. Taha and M. J. Ablowitz, J. Comput. Phys. 55, 203 (1984).

[37] H. A. Rose and M. I. Weinstein, Physica D 30, 207 (1988). 